\title{
Influenza vaccination in HIV-infected patients
}

\author{
Michał Jędrychowski ${ }^{1}$, Jacek Czepiel ${ }^{1}$, Mateusz Michalak², Aleksander Garlicki ${ }^{1}$ \\ ${ }^{1}$ Department of Infectious and Tropical Diseases, Jagiellonian University, Medical College, Krakow, Poland \\ ${ }^{2}$ Students' Scientific Society, Jagiellonian University Medical College, Krakow, Poland
}

\begin{abstract}
Introduction: One of the most serious threats for people infected with human immunodeficiency virus (HIV) is the risk of influenza co-infection and complications thereof. Not only is this population fundamentally more susceptible to flu but also prolonged replication and excretion of the virus, longer illness period, higher complication rate, flu-associated mortality, and risk of cardiovascular disease have all been noted.

Material and methods: The aim of our study was to assess vaccine efficacy against influenza in HIV-infected patients in various stages of the disease in comparison to a control group and estimate the influence of the vaccine on respiratory system infection rates. We prospectively studied 78 patients. Our study group included 47 patients with HIV and 31 healthy volunteers. The participants were immunised with TIV (trivalent influenza vaccine). Humoral response as an anti-AH1N1 (A/Brisbane/59/07), -AH3N2 (A/Brisbane/10/07), and -B strain (B/Florida/4/06) haemagglutinin antibody titre was measured. The assay was performed twice: before administration of the vaccine and a month after.

Results: The HIV-infected group exhibited a weaker immune response than the control group; however, the immunisation did provide partial protection against influenza. Vaccine efficacy was similar, regardless of CD4 count. Trivalent influenza vaccine successfully prevented influenza-associated bacterial pneumonia.

Conclusions: The study demonstrates that routine vaccination against influenza in HIV-infected patients, regardless of immune system deficiency, is substantiated.

HIV AIDS Rev 2019; 18, 1: 44-49 DOI: https://doi.org/10.5114/hivar.2019.84236
\end{abstract}

Key words: HIV, influenza, vaccination, response rate, protection rate.

\section{Introduction}

Human immunodeficiency virus (HIV) infection and acquired immunodeficiency syndrome (AIDS) remains a paramount public health issue across the population, in its course leading to a progressive immune deterioration, opportunistic infections, and malignancies. Since the introduc-

Address for correspondence: Dr. Michał Jędrychowski, Department of Infectious and Tropical Diseases, Jagiellonian University, Medical College, 5 Śniadeckich St., 31-531 Kraków, Poland, e-mail: mjedry@gmail.com

tion of highly active antiretroviral drugs, patients' survival rates have markedly increased. Nevertheless, one of the serious threats to the population of HIV-infected patients remains the risk of influenza infection and complications thereof, consisting notably of bacterial pneumonia [1]. Not only are HIV-infected people more susceptible to influen$\mathrm{za}$, but also prolonged replication and excretion of the virus,

Article history:

Received: 07.01.2019

Received in revised form: 05.02.2019

Accepted: 05.03.2019

Available online: 28.03.2019
International Journal of HIV-Related Problem

HIV \& AIDS

R e vi e w 
longer illness period, higher complication rate, flu-associated mortality, and risk of cardiovascular disease have all been noted in that population [2-4]. Lin et al. demonstrated that in a group of patients with AIDS, the flu-associated mortality rate is far higher (94-146/100 000) than in the general population (0.9-1.0/100 000 for ages 25-45 years and 64-70/100 000 for ages 65 years and over). It has also been shown that the risk of hospitalisation caused by a respiratory/cardiovascular condition in HIV-infected women is markedly higher during the flu season $[5,6]$. Additional factors in a portion of the population, such as smoking, use of inhaled narcotics, and chronic respiratory diseases increase the risk of infection even more [7].

Vaccination is one of the classic methods of preventing infection. Vaccinating people with impaired immunity, regardless of the cause of impairment, presents two basic problems: the first is the possibility of adverse effects following vaccination. In HIV-infected patients, particularly with CD4 count below $200 / \mu \mathrm{l}$, attenuated ("live") vaccines should not be used because of the immune deficiency. Live vaccines against cholera, typhoid fever, flu, tuberculosis, polio (OPV), and smallpox are contraindicated regardless of CD4 count. Killed vaccines, however, are as safe for immunodeficient patients as they are for the immunocompetent. The second problem is an insufficient or even non-existent response to vaccination, which, again, stems from the impairment to the immune system. In HIV-infected patients it is recommended to defer use of inactivated vaccines until the CD4 count exceeds $200 / \mu \mathrm{l}[1]$.

The aim of our study was to assess vaccine efficacy against influenza in HIV-infected patients in various stages of the disease in comparison to a control group and estimate the influence of the vaccine on respiratory system infection rates.

\section{Material and methods}

\section{Patients}

We prospectively studied 78 patients. Our study group consisted of 47 patients: 11 females (23.4\%) and 36 males (76.6\%), aged between 19 and 52 years (age average: 36.3 years) with HIV, treated at the Infectious and Tropical Diseases Department, University Hospital in Krakow, and 31 healthy volunteers: 10 females (32.3\%) and 21 males (67.8\%) aged 22 to 50 years (average: 26.5 years). The diagnosis of HIV infection was based on the detection of HIV-specific antibodies with an ELISA test (Hoffmann-La Roche Ltd., Basel, Switzerland), confirmed by a Western-blot (INNO-LIA, Fujirebio Inc., Pennsylvania, US). The HIV-infected group was divided into two subgroups: one with CD4 count under $350 / \mu \mathrm{l}$ and the second over $350 / \mu \mathrm{l}$. The exclusion criteria were: contraindications to the influenza vaccine, autoimmune disorders, diseases associated with immunosuppression, diabetes, and, in the control group, HIV infection.

\section{Vaccination}

Participants were vaccinated with an inactivated vaccine (TIV, trivalent influenza vaccine), consistent with WHO recommendations for the 2008/2009 flu season (the study was conducted in 2008/2009). Fluarix, a GlaxoSmithKline product, was used. Subsequently, humoral response as an antiAH1N1 (A/Brisbane/59/07), -AH3N2 (A/Brisbane/10/07), and $-\mathrm{B}$ strain $(\mathrm{B} /$ Florida/4/06) haemagglutinin antibody titre was measured. The measurement was taken twice: before administration of the vaccine and a month after.

\section{Blood tests}

Blood samples were collected in the morning (from 7.00 a.m. to 8.00 a.m.), centrifuged ( $3500 \mathrm{~g} / 15 \mathrm{~min})$, and stored at $-80^{\circ} \mathrm{C}$ until assayed. Analysis of CD4+ and CD8+ lymphocyte subpopulations in the blood was performed with the FACSCalibur flow cytometry platform (BD Biosciences, New Jersey, US). Quantitative measurement of HIV RNA in patients' serum was performed in vitro with the COBAS TaqMan 48 analyser (Hoffmann-La Roche Ltd., Basel, Switzerland) as a nucleic acid amplification test. This assay, through real-time PCR technology, makes it possible to quantify HIV-1 RNA with a linear range of 47-10 000000 copies/ml.

TIV efficacy was expressed as a defined immune response: anti-haemagglutinin titre against administered vaccine antigens. Antibody concentration was obtained through a haemagglutination inhibition assay (according to WHO guidelines), using a $0.75 \%$ turkey RBC solution and the recommended viral strains, manufactured in fertilised chicken eggs or cell cultures. Based on the obtained data, parameters of vaccine efficacy were established: protection rate (PR, the percentage of people who produced an antibody titre of at least 40), geometric mean titre (GMT), response rate (RR, the percentage of people to exhibit an at least two-fold increase in antibody titre), and mean fold increase (MFI, a geometric mean of the fold change before and after vaccination).

The study was conducted in accordance with the Declaration of Helsinki (1975) and was approved by the local Ethics Committee.

\section{Statistical methods}

Analysis of the data was conducted using parametric tests. Student's $t$-test was employed to compare the antibody titres in the analysed groups, and ANOVA to compare the HIV-infected subgroups with the control group and each other before and after the vaccination and to compare the response to each influenza antigen. Statistical analysis was performed using Statistica 10 software (StatSoft ${ }^{\circledR}$ Inc., USA). Statistical hypothesis testing yielded values of $p<0.05$. The study was conducted in accordance with the Declaration of Helsinki (1975) and approved by the Jagiellonian University Ethics Committee. All study participants signed an informed consent form. 
Table 1. Mean titres of anti-haemagglutinin antibodies for the AH1N1, AH3N2, and B strains in HIV-infected patients and the control group before immunisation and one month after

\begin{tabular}{|c|c|c|c|c|}
\hline \multirow{2}{*}{ Antibody type } & \multicolumn{2}{|c|}{ Control group $(n=31)$} & \multicolumn{2}{|c|}{ HIV-infected patients $(n=47)$} \\
\hline & Antibody titre (mean \pm SD) & $p$ & Antibody titre (mean \pm SD) & $p$ \\
\hline Anti-H1 before immunisation & $18.1 \pm 56.5$ & \multirow{2}{*}{0.001} & $8.1 \pm 11.4$ & \multirow{2}{*}{0.001} \\
\hline Anti-H1 after one month & $315.5 \pm 450.3$ & & $185.1 \pm 361.1$ & \\
\hline Anti-H3 before immunisation & $35.6 \pm 115.8$ & \multirow{2}{*}{0.001} & $10.7 \pm 16.6$ & \multirow{2}{*}{0.006} \\
\hline Anti-H3 after one month & $320.8 \pm 464.6$ & & $121.70 \pm 273.9$ & \\
\hline Anti-B before immunisation & $10.9 \pm 14.9$ & \multirow{2}{*}{0.004} & $9.6 \pm 23.1$ & \multirow{2}{*}{$<0.001$} \\
\hline Anti-B after one month & $75.9 \pm 119.3$ & & $39.3 \pm 68.5$ & \\
\hline
\end{tabular}

Table 2. Mean titres of anti-haemagglutinin antibodies for the AH1N1, AH3N2, and B strains in HIV-infected patients before immunisation and one month later in relation to CD4 count

\begin{tabular}{|c|c|c|c|c|}
\hline \multirow[t]{2}{*}{ Antibody type } & \multicolumn{2}{|c|}{$\begin{array}{l}\text { HIV-infected patients, CD4 }<350 / \mu \mathrm{l} \\
\qquad(n=29)\end{array}$} & \multicolumn{2}{|c|}{ 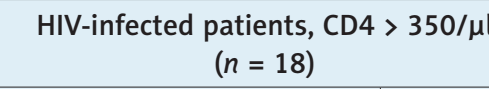 } \\
\hline & Antibody titre (mean \pm SD) & $p$ & Antibody titre (mean \pm SD) & $p$ \\
\hline Anti-H1 before immunisation & $6.6 \pm 4.0$ & \multirow{2}{*}{0.03} & $10.6 \pm 17.7$ & \multirow{2}{*}{0.03} \\
\hline Anti-H1 after one month & $149.3 \pm 328.1$ & & $242.8 \pm 412.1$ & \\
\hline Anti-H3 before immunisation & $11.7 \pm 20.0$ & \multirow{2}{*}{0.03} & $9.2 \pm 9.1$ & \multirow{2}{*}{0.01} \\
\hline Anti-H3 after one month & $117.2 \pm 261.8$ & & $128.9 \pm 299.9$ & \\
\hline Anti-B before immunisation & $10.7 \pm 28.7$ & \multirow{2}{*}{0.02} & $7.8 \pm 8.8$ & \multirow{2}{*}{0.002} \\
\hline Anti-B after one month & $43.6 \pm 83.6$ & & $32.2 \pm 32.9$ & \\
\hline
\end{tabular}

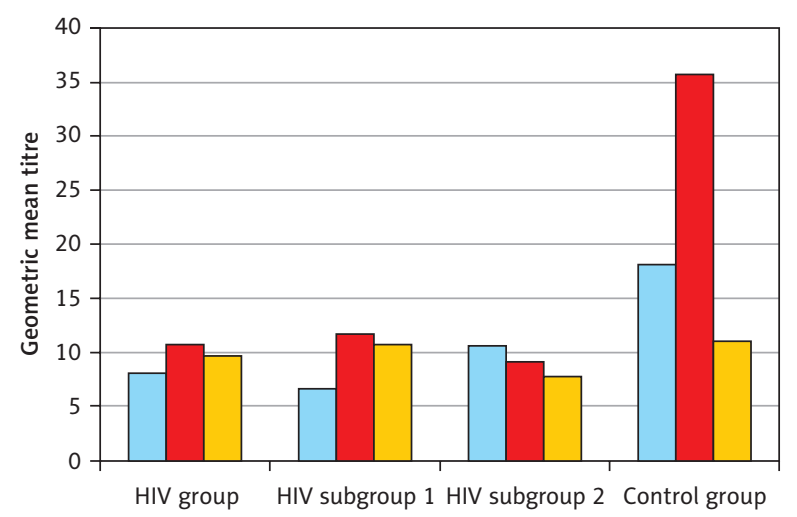

$\sqcap$ Before vaccination $\mathrm{H} 1 \quad \square$ Before vaccination $\mathrm{H} 3 \quad \square$ Before vaccination B

Figure 1. Antibody titre in each group before immunisation

\section{Results}

\section{Vaccine efficacy in the studied groups}

Both groups were demonstrated to show significantly higher anti-haemagglutinin antibody titre values one month after vaccination in comparison to the titre before immunisation. TIV was efficacious for the whole analysed population.

It was shown that anti-haemagglutinin specific antibody titre values were significantly higher one month after vacci- nation in both groups. TIV in the HIV-infected patient group with CD4 count below 350/ $\mu$ l was efficacious as well.

\section{Disparity in protective antibody titres between the HIV-group and the control group before and after immunisation}

Differences in antibody titre between the analysed groups were compared before administering the vaccine. Although titres of all the tested antibodies were noticeably higher in the control group in comparison to the HIV-infected group, the differences were not statistically significant (Fig. 1). A similar comparison was made of the titre in the analysed groups one month after immunisation. The mean antibody titre against AH3N2 after immunisation in the HIV-infected group was significantly lower than in the control group. The rest of the range did not show statistical significance (Fig. 2).

\section{Response to vaccine antigens in the HIV-infected group}

In the HIV-infected patient group average values of anti$\mathrm{H} 1,-\mathrm{H} 3$, and -B anti-haemagglutinin titre values were varied. The strongest response observed was to the AH1N1 strain and the weakest for B/Florida/4/06. A statistically significant difference, however, was only observed between antigen AH1N1 and B antibody titre $(p=0.008)$ (Fig. 3). 


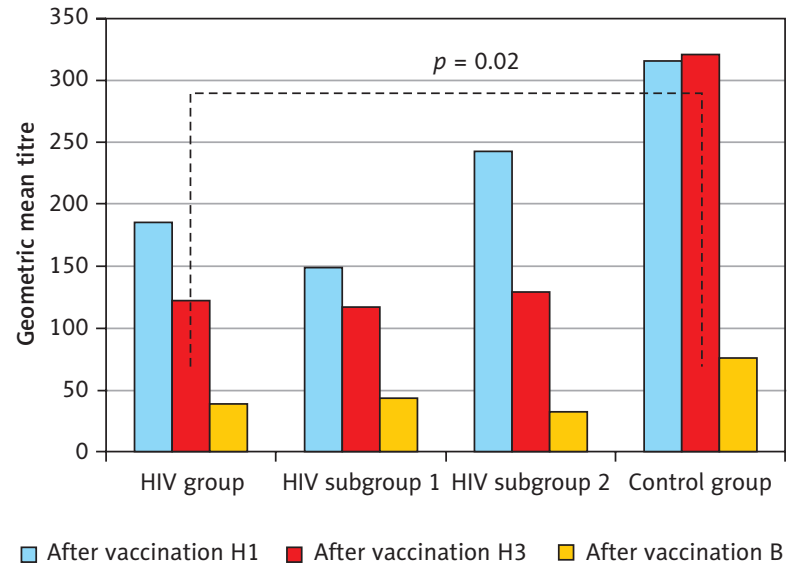

Figure 2. Antibody titre in each group after immunisation

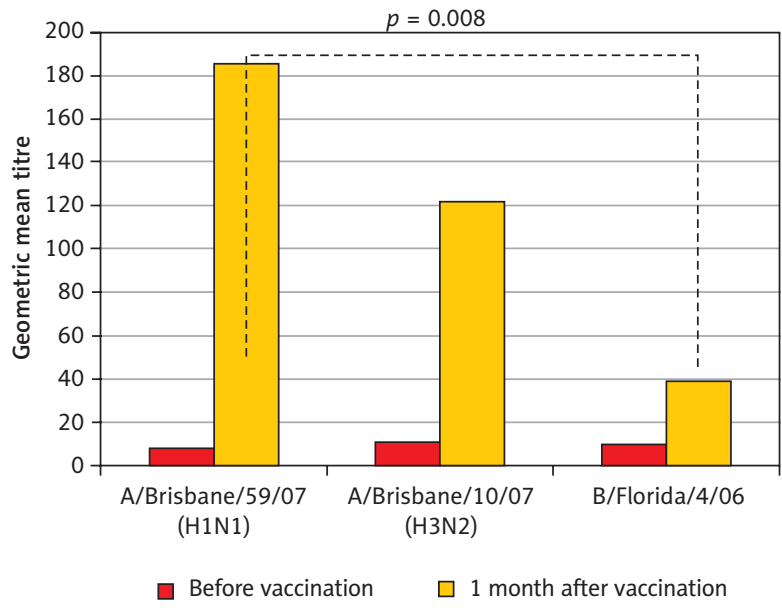

Figure 3. Response to vaccine antigens in HIV-infected patients

Table 3. Parameters of influenza vaccine efficacy in studied groups

\begin{tabular}{|c|c|c|c|c|c|c|c|}
\hline \multirow[b]{2}{*}{ Antigen } & \multirow[b]{2}{*}{ Group } & \multicolumn{2}{|c|}{ GMT } & \multirow{2}{*}{$\begin{array}{c}\text { MFI } \\
\begin{array}{c}\text { One month } \\
\text { after } \\
\text { immunisation }\end{array}\end{array}$} & \multicolumn{2}{|c|}{ PR [\%] } & \multirow{2}{*}{$\begin{array}{c}\text { RR [\%] } \\
\text { One month } \\
\text { after } \\
\text { immunisation }\end{array}$} \\
\hline & & $\begin{array}{c}\text { Before } \\
\text { immunisation }\end{array}$ & $\begin{array}{l}\text { One month } \\
\text { after } \\
\text { immunisation }\end{array}$ & & $\begin{array}{c}\text { Before } \\
\text { immunisation }\end{array}$ & $\begin{array}{c}\text { One month } \\
\text { after } \\
\text { immunisation }\end{array}$ & \\
\hline \multirow{2}{*}{$\begin{array}{l}\text { A/Brisbane/59/07 } \\
\text { (H1N1) }\end{array}$} & HIV & 6.2 & 35.0 & 5.6 & 2.1 & 51.1 & 57.5 \\
\hline & Control & 7.3 & 111.9 & 15.3 & 6.5 & 83.9 & 74.2 \\
\hline \multirow{2}{*}{$\begin{array}{l}\text { A/Brisbane/10/07 } \\
\text { (H3N2) }\end{array}$} & HIV & 6.9 & 27.3 & 3.9 & 10.6 & 44.7 & 48.9 \\
\hline & Control & 9.8 & 83.7 & 8.6 & 16.1 & 74.19 & 67.7 \\
\hline \multirow{2}{*}{ B/Florida/4/06 } & HIV & 5.9 & 15.1 & 2.6 & 4.3 & 36.2 & 38.3 \\
\hline & Control & 7.5 & 32.7 & 4.4 & 6.5 & 54.8 & 54.8 \\
\hline
\end{tabular}

$G M T$ - geometric mean titre, $M F I$ - mean fold increase, $P R$ - protection rate, $R R$ - response rate

\section{Calculation of the parameters conductive to the assessment of vaccine efficacy}

The values of the first two parameters (PR and GMT) may be overestimated, since a fraction of the patients had already been immunised (seropositive before vaccination). The last two parameters, namely RR and MFI, account for the change dynamic, but may in turn underestimate the results [8]. The parameters used to evaluate the influenza vaccination efficacy are presented in Table 3.

\section{Incidence of sequelae bacterial respiratory infections}

Based on patients' medical records, no complications in the form of bacterial respiratory tract infections (pneumoniae) were found.

\section{Discussion}

Yearly vaccination is the most effective method of preventing influenza and its complications. The most commonly used vaccines are inactivated and consist of three viruses: two different influenza type A strains and one influenza type B strain. Due to high mutation rate of the virus, the WHO issues annual recommendations for influenza vaccine formulations before every flu season. The markers of post-immunisation protection are anti-haemagglutinin and virus-neutralising antibody titres in the serum. An increase in antibody titre post-vaccination lowers the risk of disease caused by strains similar to those included in the vaccine. Parameters such as PR (usually defined as haemagglutination titre of $1: 32$ or $1: 40$ ) correlate well with immunity at a population level $[9,10]$.

According to the requirements of the Commission of the European Communities and the Committee for Proprietary Medicinal Products for influenza vaccination for people aged 18-60 years, average antibody level MFI should be $\geq 2.5, P R \geq 70 \%$, and $R R \geq 40 \%$. In our study the immunisation induced a significant titre of anti-haemagglutinin antibodies in all the participants. In the control group, the increase in antibody level averaged from 4.3 (B strain) through 8.6 (AH1N2) to 15.3 (AH1N1). PR > 70\% was achieved for the $\mathrm{AH} 1 \mathrm{~N} 1$ and $\mathrm{AH} 3 \mathrm{~N} 2$ strains; only for the B strain was 
it $54.8 \%$. Response rates fluctuated between $54.8 \%$ and $74.1 \%$. In a healthy population the vaccine is usually highly immunogenic and effective, which proved to be true in our study as well. The HIV-infected patients exhibited a weaker response to the immunisation. Antibody GMT after vaccination was 2-3 times lower than in the control group; statistical significance, however, was shown only in the response to AH3N2 antigen. Despite that, MFI exceeded the recommended level of 2.5 for all the strains. PR fluctuated between $36.1 \%$ (B strain) and 51\% (AH1N1 strain) and did not reach the desirable level of $70 \%$. RR exceeded $40 \%$ for the first two strains, and for the B strain it was $38.2 \%$, essentially meeting the requirements [11-14].

Analysis of studies comparing incidence rates of influenza among vaccinated and unvaccinated HIV-infected patients has indicated a moderate advantage of vaccinating [3, 15-17]. Mahdi et al. conducted an extensive, randomised, double-blind trial among HIV-infected citizens of Johannesburg, South Africa. It yielded a percentage of seroconversion of $52.6-60.8 \%$. The average increase in antibody concentration ranged from 4.1-10.2, which was higher than in our study. Efficacy in preventing laboratory-confirmed influenza was high and reached $75.5 \%$ [18]. Atashili et al. conducted a meta-analysis of four studies, encompassing $646 \mathrm{HIV}$-infected patients in total, and found TIV to be moderately efficacious in limiting the influenza incidence rate [19]. The only study on an adult HIV-infected population in Poland involved 34 patients. PR after a month ranged from 18\% (AH1N1 strain) through 41\% (B strain) to $79 \%$ (AH3N2 strain). However, the value for the latter has been overestimated due to a high percentage (50\%) of patients with a pre-existing high protective antibody titre. It stemmed from prior contact with said subtype, which dominated at that time in Poland. Antibody GMT increased by a factor of 1.5-5.5, which is comparable to our results, yielding an increase by a factor of 2.6-5.6. Like here, no statistically significant difference in humoral response between patients with different CD4 values nor between patients in different clinical stages of the disease was shown [20].

CD4 count is a key factor in immune system function of HIV-infected patients. As the disease progresses, the B memory cell function declines, which is particularly evident in patients with high viral load. Hence, they exhibit a weaker humoral response to immunisation. It has been established that the independent factors correlated with post-vaccination response strength are CD4 count and viral load [21, 22]. Low CD 4 count, especially $<200 / \mu l$, reduces vaccine efficacy in general and similarly for the influenza vaccine [23-25]. It has been shown that in patients with high CD4 count the immunisation induced a high specific protective antibody titre $[23,24,26]$. Patients in the AIDS phase, in turn, often did not achieve a protective antibody titre $[23,24]$. Moreover, administration of a second dose of the vaccine did not improve the response $[23,27]$. In a randomised trial in children with average CD4 count above $400 / \mu \mathrm{l}$, immunisation was efficacious in preventing laboratory-confirmed influenza at a similar rate as in the general population [28]. Fine et al. showed efficacy only in patients whose CD4 count exceeded $100 / \mu$ l or whose viral load was under $30000 / \mathrm{ml}$ [3]. Furthermore, patients undergoing antiretroviral therapy (ART) longer than three months, with a CD4 count increase of at least $15 \%$ and viral load decrease to under 1000 copies $/ \mathrm{ml}$, respond better to treatment. An additional benefit of ART is an increase in concentration of specific antibodies left over from prior immunisations when no booster dose has been given. It has been shown that immunogenicity of the influenza vaccine is higher in patients successfully treated with ART and without progression of the disease [29]. Our study did not find such a relation. TIV was efficacious for patients

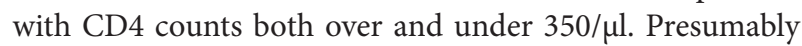
application of a different patient distribution (with a significantly lower CD4 count) would show an impact of a nominal CD4 cell level on vaccine efficacy in said groups.

Influenza, in contrast to many diseases entirely preventable with immunisation, is very common with a high incidence rate worldwide. That poses a challenge for studies on vaccine immunogenicity because a sizeable portion of the studied population may already have a protective antibody titre from prior contact with a given virus upon entrance into the study. In our study, the geometric mean of antibody titres was low and in HIV-infected patients was in the range of 5.97 to 6.24 , and in the control group 7.31 to 9.77 . No statistically significant difference between the groups was shown. This proves that most patients had not had prior contact with viral antigens corresponding to vaccine antigens.

Inactivated influenza vaccines available in Poland contain three flu strain antigens. One element of the study was an assessment of the response to individual antigens in HIV-infected patients, where a weaker response was observed to the B strain. This tendency has not been backed by any of the quoted publications by other authors and possibly stems from a weaker immunogenicity of the antigen used in said flu season.

In a typical flu season in Poland (autumn to early spring months) we observe raised mortality and hospitalisation rates during the circulation of the virus. Based on the correlation between influenza activity and seasonality of pneumococcal pneumonia, one could infer that in some patients admitted for invasive pneumococcal pneumonia we might expect a comorbid influenza infection [30]. The unnecessary deaths and admissions in flu season, which are, at least partly, caused by influenza infection, fit into a broad category of respiratory and circulatory hospitalisations [31, 32]. In our study we analysed medical records of HIV-infected patients, finding no instances of bacterial respiratory infections throughout nine months post-vaccination. We can therefore speculate that TIV in HIV-infected patients successfully prevents complications in the form of pneumonia. A meta-analysis of 15 cohort studies and clinically controlled trials evaluating the factual effectiveness of TIV in preventing flu/pneumonia-associated hospitalisations in patients above 65 years old showed that TIV use helped avert $6-26 \%$ of doc- 
tor visits and reduced the relative risk of death due to flu or pneumonia [33].

In conclusion, immune response to influenza vaccination was weaker in HIV-infected patients than in the healthy population; nevertheless, the immunisation did provide partial protection against the flu. Vaccine efficacy was comparable regardless of CD4 count. Immunisation successfully prevented flu-associated bacterial pneumonia. The study demonstrates that routine vaccination against influenza in $\mathrm{HIV}$-infected patients, regardless of immune system deficiency, is substantiated.

\section{Conflict of interest}

The authors declare no potential conflicts of interest with respect to the research, authorship, and/or publication of this article.

\section{References}

1. Trovato M, D'Apice L, Prisco A, de Bernardinis P. HIV vaccination: a roadmap among advancements and concerns. Int J Mol Sci 2018; 19: E1241.

2. Zanetti AR, Amendola A, Besana S, et al. Safety and immunogenicity of influenza vaccination in individuals infected with HIV. Vaccine 2002; 20: B29-32.

3. Fine AD, Bridges $\mathrm{CB}$, De Guzman AM, et al. Influenza A among patients with human immunodeficiency virus: an outbreak of infection at residential facility in New York City. Clin Inf Dis 2001; 32: 1784-1791.

4. Radwan HM, Cheeseman SH, Lai KK, et al. Influenza in human immunodeficiency virus-infected patients during the 1997-1998 influenza season. Clin Infect Dis 2000; 31: 604-606.

5. Lin JC, Nichol KL. Excess mortality due to pneumonia or influenza during influenza seasons among persons with acquired immunodeficiency syndrome. Arch Intern Med 2001; 161: 441-446.

6. Neuzil KM, Reed GW, Mitchel EF Jr, et al. Influenza-associated morbidity and mortality in young and middle-aged women. JAMA 1999; 281: 901-907.

7. Conley LJ, Bush TJ, Buchbinder SP, et al. The association between cigarette smoking and selected HIV-related medical condictions. AIDS 1996; 10: 1121-1126.

8. Beyer WEP, Palache AM, Lüchters G, et al. Seroprotection rate, mean fold increase, seroconversion rate: which parameter adequately express seroresponse to influenza vaccination? Virus Res 2004; 103: 125-132.

9. Esposito S, Franco E, Gavazzi G, et al. The public health value of vaccination for seniors in Europe. Vaccine 2018; 36: 2523-2528.

10. Hirota Y, Kaji M, Ide S, et al. Antibody efficacy as a keen index to evaluate influenza vaccine effectiveness. Vaccine 1997; 15: 962-967.

11. Brydak LB. Pandemic of flu - myth or real threat? Oficyna Wydawnicza RYTM, Warszawa 2008.

12. Commission of the European Communities. The rules governing medicinal products in the European Community. Commission Eur Commun 1992; 3: 93-98.

13. Committee for Proprietary Medicinal Products (CPMP). Note for guidance on harmonisation of requirements for influenza vaccines (revision). 1997: CPMP/BWP/214/96.

14. Brydak LB, Białek J, Rudnicka H, et al. Seroconversion assessment in billeted Military Medical University student group after anti influenza subunit vaccinations in 1993/1994 in Poland. Antiinfect Drugs Chemother 1997; 15: 13-16.
15. Anema A, Mills E, Montaner J, et al. Efficacy of influenza vaccination in HIV-positive patients: a systemic review and meta-analysis. HIV Med 2008; 9: 57-61.

16. Tasker SA, Treanor JJ, Paxton WB, et al. Efficacy of influenza vaccination in HIV-infected persons. A randomized, double-blind, placebo-controlled trial. Ann Intern Med 1999; 131: 430-433.

17. Yamanaka H, Teruya K, Tanaka M, HIV/Influenza Vaccine Study Team, et al. Efficacy and immunologic responses to influenza vaccine in HIV-1-infected patients. J Acquir Immune Defic Syndr 2005; 39: 167-173.

18. Madhi SA, Maskew M, Koen A, et al. Trivalent inactivated influenza vaccine in African adults infected with human immunodeficient virus: double blind, randomized clinical trial of efficacy, immunogenicity, and safety. Clin Infect Dis 2011; 52: 128-137.

19. Atashili J, Kalilani L, Adimora AA. Efficacy and clinical effectiveness of influenza vaccines in HIV-infected individuals: a metaanalysis. BMC Infect Dis 2006; 6: 138.

20. Brydak LB, Hryniewicz HJ, Machala M, et al. Humoral response to influenza vaccination in HIV-infected patients. Clin Drug Invest 1999; 17: 441-449.

21. Amu S, Ruffin N, Rethi B, et al. Impairment of B-cell functions during HIV-1 infection. AIDS 2013; 27: 2323-2334.

22. Rubin LG, Levin MJ, Ljungman P, Infectious Diseases Society of America, et al. 2013 IDSA clinical practice guideline for vaccination of the immunocompromised host. Clin Infect Dis 2014; 8: e44-100.

23. Kroon FP, van Dissel JT, de Jong JC, et al. Antibody response after influenza vaccination in HIV-infected individuals: a consecutive 3-year study. Vaccine 2000; 18: 3040-3049.

24. Staprans SI, Hamilton BL, Follansbee SE, et al. Activation of virus replication after vaccination of HIV-1-infected individuals. J Exp Med 1995; 182: 1727-1737.

25. Cooper C, Hutton B, Fergusson D, et al. A review of influenza vaccine immunogenicity in HIV-infected adults. Can J Infect Dis Med Microbiol 2008; 19: 419-423.

26. Huang KL, Ruben FL, Rinaldo CR Jr, et al. Antibody responses after influenza and pneumococcal immunization in HIV infected homosexual men. JAMA 1987; 257: 2047-2050.

27. Miotti PG, Nelson KE, Dallabetta GA, et al. The influence of HIV infection on antibody responses to a two-dose regimen of influenza vaccine. JAMA 1989; 262: 779-783.

28. Chadwick EG, Chang G, Decker MD, et al. Serologic response to standard inactivated influenza vaccine in human immunodeficiency virus infected children. Pediatr Infect Dis J 1994; 13: 206-211.

29. Crum-Cianflone NF, Eberly LE, Duplessis C, et al. Immunogenicity of a monovalent 2009 influenza A (H1N1) vaccine in an immunocompromised population: a prospective study comparing HIV-infected adults with HIV-uninfected adults. Clin Infect Dis 2011; 52: 138-146.

30. Walter ND, Taylor TH, Shay DK, Thompson WW, Active Bacterial Core Surveillance Team, et al. Influenza circulation and the burden of invasive pneumococcal pneumonia during a non-pandemic period in the United States. Clin Infect Dis 2010; 50: 175-183.

31. Thompson WW, Shay DK, Weintraub E, et al. Influenza-associated hospitalizations in the United States. JAMA 2004; 292: 1333-1340.

32. Thompson WW, Shay DK, Weintraub E, et al. Mortality associated with influenza and respiratory syncytial virus in the United States. JAMA 2003; 289: 179-186.

33. Vu T, Farish S, Jenkins $M$, et al. A meta-analysis of effectiveness of influenza vaccine in person aged 65 years and over living in the community. Vaccine 2002; 20: 1831-1836. 